\title{
Use of a Long Term Ventricular Assist Device with a Modified Circuit and Modified Care Model for Short Term Extracorporeal Support: An Institutional Experience
}

\author{
Tanya Chadha*, Shamsur Chowdhury, Eric Ceithaml and Michael Shillingford \\ Wolfson Children's Hospital, Jacksonville, FL, USA
}

*Corresponding author: Chadha Tanya, Wolfson Children's Hospital, Jacksonville, FL, USA

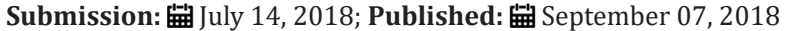

\section{Introduction}

The industry of supportive mechanical devices has the pressure of needing to produce products that are reliable, durable, cost effective, and require low maintenance and oversight. In the pediatric population, mechanical support for patients with congenital heart disease is an ongoing challenge. Here we briefly describe our institution's experience in providing short term extracorporeal support for pediatric congenital heart disease patients through innovative modifications to traditional extracorporeal circuitry and strategies.

Monge and colleagues at Ann \& Robert H. Lurie Children's Hospital in Chicago describe the use of a continuous flow extracorporeal Ventricular Assist Device (VAD) with modifications (variable restrictive recirculation shunt, oxygenator) to support univentric ular and biventricular patients as a bridge to heart transplant. This group cites "availability, low prime volume, greater patient flow range, and lower cost" as reasons that this modified VAD system could be considered as an alternative to extracorporeal membrane oxygenation (ECMO) in the pediatric population. We chose to employ the Tandem Heart VAD (Cardiac Assist, Pittsburgh, PA) with further circuit modifications (hemoconcentrator in the recirculation shunt, Figure 1) as well as modifications in the traditional ECMO team model and in circuit management strategies in our institution's pediatric cardiovascular intensive care unit as the primary mode of short term circulatory support for our congenital heart and medical cardiac patient population.

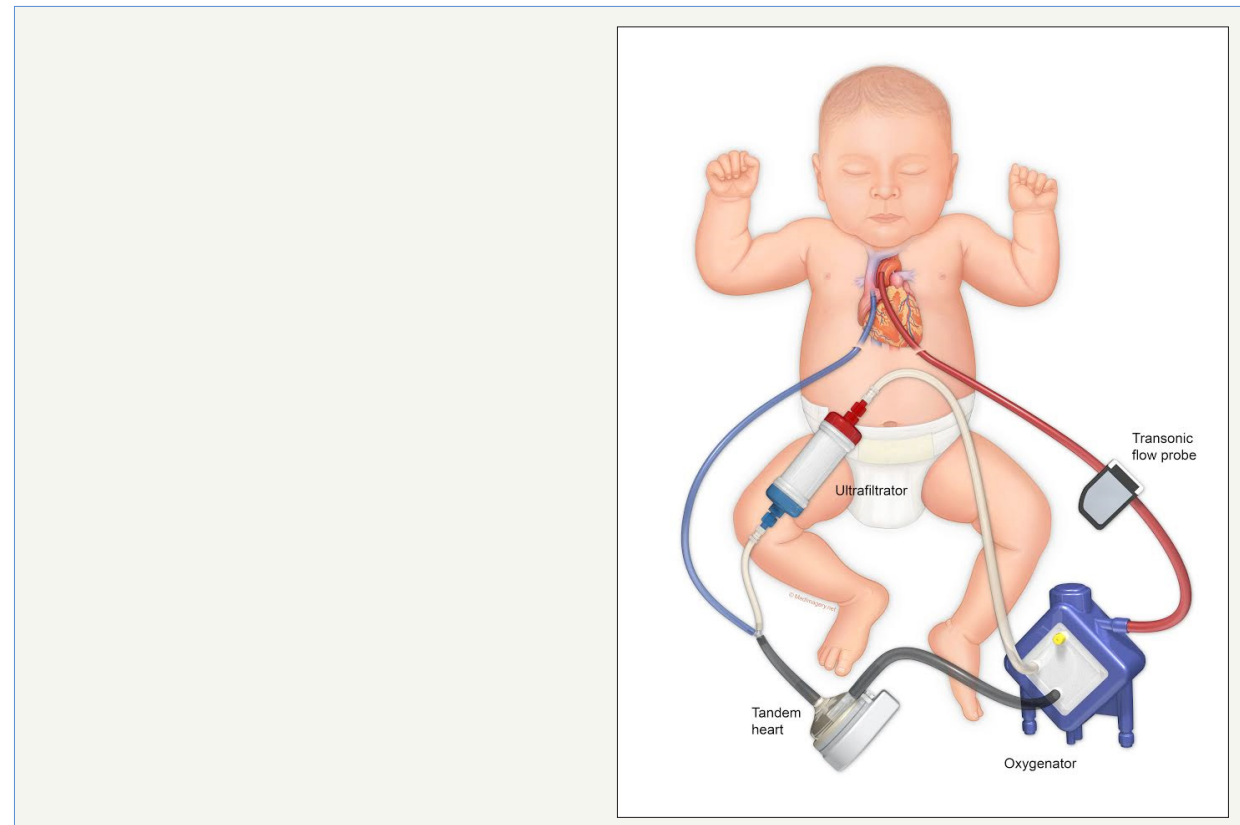

Figure 1: Modified TandemHeart circuitry. Tandem Heart VAD, Maquet Quadrox pediatric oxygenator, Sorin DHF06 hemoconcentrator for ultra filtration. 


\section{Strategies}

The same modified VAD circuit was deployed in all patients, regardless of size and physiology. Patients were cannulated via right internal jugular vein/carotid artery or centrally at the bedside or in the operating room by a team comprised of cardiovascular surgery, perfusion, cardiac nursing and cardiac critical care/ cardiac anesthesia services. Optimal flows based on clinical needs were achieved via the recirculation shunt in the circuit and "gate clamping" of arterial limb of the circuit as needed. Our circuit design minimized tubing and connections and we enforced a "closed circuit", i.e. no blood draws from or infusions into the circuit. This design and these practices were developed to minimize circuit thrombosis, to promote circuit longevity, and allowed for decreased heparin utilization which was particularly helpful in our post-op surgical population. In addition to ultrafiltration, we also were able to provide minimal dialysis when there was a clinical need by exchanging the hemoconcentrator for a dialyzer. While providing full cardiopulmonary support and continuous renal replacement therapy (CRRT) capabilities, we also adopted a low oversight deployment model and bedside model of care (1 patient: 1 nurse, simplified circuit monitoring) based around those typically employed in VAD patients. The traditional ECMO team model at our institution requires the involvement of multiple team members (primer, a specialist, and a nurse), potentially longer deployment times and a larger team for ongoing management which in turn increases cost and care complexity.

\section{Institutional Experience}

From January 1, 2017 to December 31, 2017, we successfully deployed our TandemHeart modified circuit 20 times in 14 patients for a variety of indications, including extracorporeal CPR. Patient information and basic run details are described in Table 1 . We report a $92 \%$ survival to decannulation rate with a $69 \%$ survival to discharge rate with a total of 3 major neurologic events (cerebellar hematoma, subdural hematoma, ischemic stroke) and 2 major thrombotic events (aortic thrombus, emboli to hands/feet). To the best of our knowledge, we report the smallest and youngest patients supported on this circuitry and are describing a novel means of providing short term extracorporeal support for both univentricular and biventricular cardiac patients in the setting of a modified care model. Simple in its circuitry, versatile in accommodating patient size and physiology, and conceptually cost effective, our TandemHeart modified circuit represents a combination of traditional VAD and ECMO strategies for a more efficient and durable approach to extracororporeal life support (ECLS) in the pediatric cardiac population.

Table 1: Patient information.

\begin{tabular}{|c|c|c|c|c|c|c|c|c|c|}
\hline & Runs & Age & Weight & ECLS Days & Anatomy & Indication & E-CPR & $\begin{array}{l}\text { Survival to } \\
\quad \text { DCN }\end{array}$ & $\begin{array}{l}\text { Survival to } \\
\text { D/C }\end{array}$ \\
\hline 1 & 2 & $2 \mathrm{mo}$ & $4.2 \mathrm{~kg}$ & $2 / 4$ & UV & LCOS \& heart failure, bridge to recovery & $\mathrm{x}$ & $\mathrm{x}$ & $\mathrm{x}$ \\
\hline 2 & 1 & $19 \mathrm{mo}$ & $8.8 \mathrm{~kg}$ & 2 & UV & Hypoxemia, bridge to repair & & $\mathrm{x}$ & $\mathrm{x}$ \\
\hline 3 & 1 & $10 \mathrm{mo}$ & $6.3 \mathrm{~kg}$ & 2 & UV & Hypoxemia, bridge to repair & $\mathrm{X}$ & $\mathrm{x}$ & $\mathrm{x}$ \\
\hline 4 & 1 & $2 \mathrm{wk}$ & $3.6 \mathrm{~kg}$ & 2 & BV & LCOS, bridge to recovery & & $\mathrm{x}$ & $\mathrm{x}$ \\
\hline 5 & 3 & $11 \mathrm{mo}$ & $8.4 \mathrm{~kg}$ & $7 / 2 / 1$ & UV & LCOS, bridge to recovery & & $\mathrm{x}$ & \\
\hline 6 & 1 & $7 \mathrm{wk}$ & $4.7 \mathrm{~kg}$ & 14 & BV & Sepsis, bridge to recovery & & $\mathrm{x}$ & \\
\hline 7 & 1 & $7 \mathrm{mo}$ & $9.1 \mathrm{~kg}$ & 3 & BV & Sepsis, bridge to recovery & $\mathrm{x}$ & & \\
\hline 8 & 1 & $16 y$ & $55 \mathrm{~kg}$ & 5 & BV & Heart failure, bridge to recovery & & $\mathrm{x}$ & $\mathrm{x}$ \\
\hline 9 & 1 & $18 \mathrm{mo}$ & $9.1 \mathrm{~kg}$ & 2 & BV & Trauma, bridge to recovery & $\mathrm{x}$ & $\mathrm{x}$ & $\mathrm{x}$ \\
\hline 10 & 1 & $3 w k$ & $3.5 \mathrm{~kg}$ & 6 & UV & LCOS, bridge to recovery & & $\mathrm{x}$ & $\mathrm{x}$ \\
\hline 11 & 2 & $3 y$ & $13.9 \mathrm{~kg}$ & $5 / 4$ & UV & LCOS and heart failure, bridge to recovery & & $\mathrm{x}$ & \\
\hline 12 & 1 & $5 \mathrm{wk}$ & $3.5 \mathrm{~kg}$ & 6 & UV & Heart failure, bridge to repair & & $\mathrm{x}$ & $\mathrm{x}$ \\
\hline 13 & 1 & $6 y$ & $20 \mathrm{~kg}$ & 7 & BV & LCOS, bridge to recovery & & $\mathrm{x}$ & $\mathrm{x}$ \\
\hline 14 & 3 & $\begin{array}{l}2 \mathrm{mo}(\mathrm{ex} \\
28 \mathrm{wk})\end{array}$ & $1.9 \mathrm{~kg}$ & $34 / 5 / 25$ & UV & $\begin{array}{c}\text { Hypoxemia \& LCOS, bridge to repair and } \\
\text { recovery }\end{array}$ & $\mathrm{X}$ & $\mathrm{x}$ & \\
\hline
\end{tabular}

Abbreviations: mo: Months; wk: Weeks; y: Years; UV: Univentricular; BV: Biventricular; DCN: Decannulation; D/C: Discharge 

(c) (i) Creative Commons Attribution 4.0

For possible submissions Click Here

Submit Article
RPN Research in Pediatrics \&
Neonatology
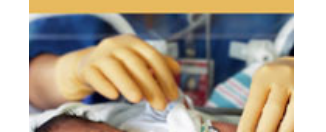

cra

- Freely accessible online immediately upon publication

- Authors retain the copyright to their work

- Licensing it under a Creative Commons license

- Visibility through different online platforms 